\title{
Investigation of Thermo Physical Properties of Liquids By Using Ultrasonic Method
}

\author{
Satheesh Kumar $\mathrm{V}^{1}$ Balamurugan $\mathrm{S}^{2}$ \\ ${ }^{1}$ Under Graduate Student, \\ ${ }^{2}$ Assistant Professor, Department of Mechanical Engineering, Coimbatore Institute of Technology, \\ Coimbatore, Tamil Nadu, India. - 641014
}

\begin{abstract}
To investigate the thermo physical properties of a liquid by varying temperature without altering its chemical composition. Physical properties influence the characteristics of the liquid. The main aim of this research was to determine the thermal conductivity of the liquids such as water, ethylene glycol, glycerin and benzene by using ultrasonic method and compare it with the standard values. These selected liquids are more commonly used as an additional agent in the already existing base liquid to enhance the heat transfer capabilities and also as base liquids in some applications. The thermal conductivity of these liquids greatly influences their usage in different applications. Basically their thermo physical properties are function of temperature, pressure, density and volume. Ultrasonic Interferometer was used to investigate the thermal conductivity, inter molecular free length and specific heat capacity by means of various theoretical relations. This ultrasonic method involving experimental setup makes use of $10 \mathrm{cc}$ constant volume liquid sample taken for each analysis. It has been found that the frequency of ultrasonic waves ( $2 \mathrm{MHz}$ and $4 \mathrm{MHz}$ ) also influence the thermal conductivity and it has been found that thermal conductivity increases with increasing frequency of ultrasonic waves. The experiment is carried out with different temperature and obtained wavelength is substituted to find the velocity of sound. Thermal conductivity obtained from these velocities using Bridgman relation coincides nearly with standard values, and the analysis is extended to find the free length and specific heat capacity using Schaaff relation. The conclusion of this research is that one thermo physical property can be theoretically determined by relation with other thermo physical properties.
\end{abstract}

Keywords: Thermal conductivity, ultrasonic interferometer, Intermolecular free length, specific heat.

\section{Introduction}

The liquids are generally used in heat transfer applications like power generation, chemical plants, automobiles, air conditioning and refrigeration systems. These applications required liquids with high heat transfer capabilities. However their heat transfer capability was limited by their very low thermal conductivity [1]. Thus Thermal conductivity increase will automatically result in higher heat transfer rate. However, we can enhance thermal conductivity by increasing the temperature. For Example, the thermal conductivity of ethylene glycol increases up to $1.8 \%$ when the temperature is changed from $308 \mathrm{~K}$ to $328 \mathrm{~K}$ [2]. Increase of thermal conductivity with temperature is found using ultrasonic methods. The ultrasonic properties and thermal conductivity have a lot of similar peculiarities. Hence by deriving a relationship between them, one property can be used to determine the others and can also lead to the unification of all thermo physical parameters. Determination of the thermal properties of aspartic acid using the ultrasonic velocity was carried out [3] and found that collision between atoms causes decrease in thermal conductivity in liquids. By utilization of mean free path of molecules in the liquid, the intermolecular free length can be computed which was used to determine the thermal conductivity of Jatropha oil [4]. Based on the need for heat transfer fluid in concentrated solar power plants, thermal conductivity of different vegetable oils are measured using thermal probe and obtained accurate results [5]. Stability of the liquids based upon temperature and how different concentration of liquid surfactants improves stability and its effect on thermal conductivity are examined [6]. Determination of Thermal conductivity of a fluid is calculated in relation with the thermal conductivity of air [7]. Thermal conductivity of electrically conducting liquids using hot wire method were observed using a insulated layer whose effect was found to be considered as negligibly small [8]. Thermal conductivity for papaya is also found using hot wire method [9]. Another method to determine the thermal conductivity was based on the heat transfer mechanism where Bridgman has discussed an empirical relation [10], characterized by direct proportionality between thermal conductivity and sound velocity in pure liquids. The temperature coefficients of thermal conductivity and sound velocity are negative, except for anomalous liquids [11]. The effect of pressure is similar both the thermal conductivity and the sound velocity increase as the pressure grows.

Intermolecular free length is the distance between two adjacent molecules of a liquid. Free length of different liquid changes with temperature and can also be used in determining the thermal properties such as thermal conductivity and specific heat capacity. Intermolecular free length is computed using ultrasonic velocity 
by means of free length theory (FLT) and collision factor theory (CFT) [12]. Intermolecular free length can also be determined from adiabatic compressibility [13].

Acoustic properties which determine the ultrasonic velocity are adiabatic compressibility, acoustic impedance and attenuation [14]. Specific heat of a liquid determines the amount of heat required for per degree rise in temperature. It also influences the heat transfer capability [14-17].

In the present work, thermal conductivity of water, ethylene glycol, glycerin and benzene are calculated experimentally by using continuous wave ultrasonic interferometer. Thermal conductivity for $2 \mathrm{MHz}$ and $4 \mathrm{MHz}$ frequencies are determined for various temperatures ranging from $30^{\circ} \mathrm{C}$ to $60^{\circ} \mathrm{C}$. Temperature ranges considered according to their melting and boiling point of each liquids. Thermal conductivity and ultrasonic velocity for the liquid is studied by varying the frequency of the ultrasonic wave. Another thermo physical property, specific heat capacity of the liquid can be computed using the intermolecular free length, thermal conductivity and ultrasonic velocity inside the liquid by making mean free path of the liquid as it's free length.

\section{Experimental Setup}

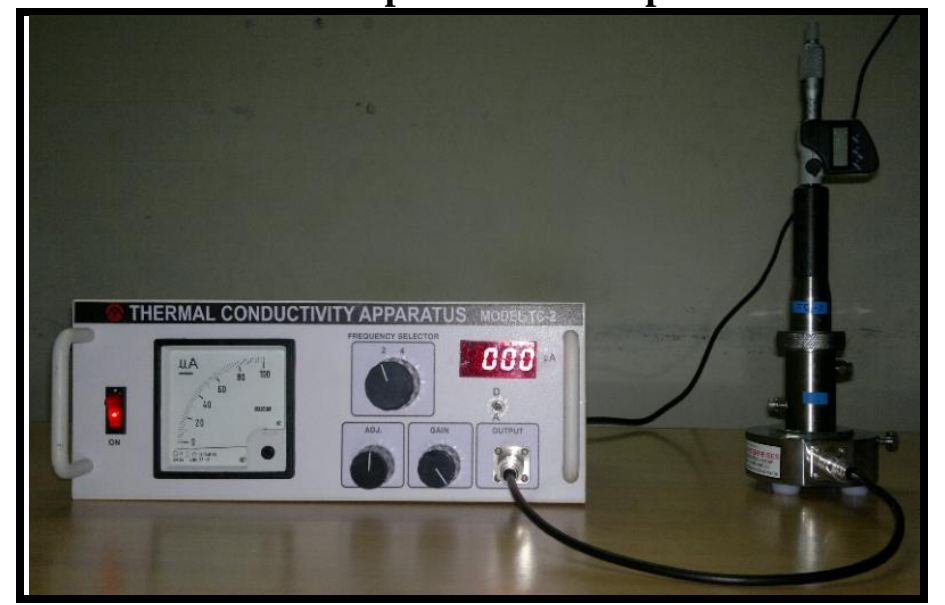

Fig 2.1 Single frequency continuous wave ultrasonic Interferometer

The experimental setup for ultrasound velocity measurement is shown in Fig. 2.1. Ultrasonic velocity measurements are carried out using Ultrasonic interferometer which yields accurate and consistent data, from which one can determine the velocity of ultrasonic sound in a liquid medium. When an ultrasonic wave propagates through a medium, the molecules in that medium vibrate over very short distance in a direction parallel to the longitudinal wave. During this vibration, momentum is transferred among molecules. This causes the wave to pass through the medium. In an ultrasonic interferometer, the ultrasonic waves are produced by the piezoelectric method. The apparatus consists of an ultrasonic cell, which is a double walled brass cell with chromium plated surfaces having a capacity of $10 \mathrm{ml}$. The double wall allows water circulation around the experimental medium to maintain it at a known constant temperature. The micrometer scale is marked in units of $0.01 \mathrm{~mm}$ and has an overall length of $25 \mathrm{~mm}$. Ultrasonic waves of known frequency are produced by a quartz crystal which is fixed at the bottom of the cell. There is a movable metallic plate parallel to the quartz plate, which reflects the waves. The waves interfere with their reflections, and if the separation between the plates is exactly an integer multiple of half-wavelengths of sound, standing waves are produced in the liquid medium. Under these circumstances, acoustic resonance occurs. The resonant waves are a maximum in amplitude, causing a corresponding maximum in the anode current of the piezoelectric generator.

If we increase or decrease the distance by exactly one half of the wavelength $(\lambda / 2)$ or an integer multiple of one half wavelength, the anode current again becomes maximum. If $d$ is the separation between successive adjacent maxima of anode current, then,

$$
\mathbf{d}=\frac{\lambda}{2}
$$

We have, the velocity ( $v$ ) of a wave is related to its wavelength $(\lambda)$ by the relation,

where $\mathbf{f}$ is the frequency of the wave.

$$
v=\lambda \mathbf{f}=\mathbf{2 d f}
$$

Ultrasonic velocities for two different frequencies $(2 \mathrm{MHz} \& 4 \mathrm{MHz})$ are calculated for various temperatures $(313 \mathrm{~K}, 318 \mathrm{~K}, 323 \mathrm{~K}, 328 \mathrm{~K}$, and $333 \mathrm{~K})$. Wavelengths obtained from the micrometer and the corresponding ultrasonic velocities were tabulated. 
Table 2.1 Ultrasonic velocity and wavelength in different liquids at different temperature for $2 \mathrm{MHz}$ and $4 \mathrm{MHz}$ frequencies

\begin{tabular}{|c|c|c|c|c|c|}
\hline Liquids & $\begin{array}{c}\text { Temperature } \\
(\mathbf{K})\end{array}$ & $\begin{array}{c}\text { Wave length } \lambda / 2 \\
(2 \mathrm{MHz})\end{array}$ & $\begin{array}{c}\text { Wave length } \lambda / 2 \\
(4 \mathrm{MHz})\end{array}$ & $\begin{array}{c}\text { Velocity of sound } v \\
(2 \mathrm{MHz})\end{array}$ & $\begin{array}{l}\text { Velocity of sound } v \\
(4 \mathrm{MHz})\end{array}$ \\
\hline \multirow{5}{*}{ Distilled Water } & 313 & 0.377 & 0.192 & 1508 & 1536 \\
\hline & 318 & 0.381 & 0.1926 & 1524 & 1540 \\
\hline & 323 & 0.386 & 0.193 & 1544 & 1547 \\
\hline & 328 & 0.387 & 0.194 & 1548 & 1552 \\
\hline & 333 & 0.388 & 0.196 & 1552 & 1568 \\
\hline \multirow{5}{*}{ Ethylene Glycol } & 313 & 0.401 & 0.203 & 1604 & 1624 \\
\hline & 318 & 0.4004 & 0.199 & 1602 & 1592 \\
\hline & 323 & 0.400 & 0.197 & 1600 & 1576 \\
\hline & 328 & 0.396 & 0.1967 & 1584 & 1573 \\
\hline & 333 & 0.390 & 0.196 & 1560 & 1568 \\
\hline \multirow{5}{*}{ Glycerin } & 313 & 0.472 & 0.2338 & 1888 & 1870 \\
\hline & 318 & 0.468 & 0.2336 & 1872 & 1868 \\
\hline & 323 & 0.462 & 0.2333 & 1848 & 1866 \\
\hline & 328 & 0.461 & 0.231 & 1844 & 1848 \\
\hline & 333 & 0.460 & 0.229 & 1840 & 1832 \\
\hline \multirow{5}{*}{ Benzene } & 313 & 0.306 & 0.175 & 1224 & 1400 \\
\hline & 318 & 0.301 & 0.171 & 1204 & 1368 \\
\hline & 323 & 0.295 & 0.170 & 1180 & 1360 \\
\hline & 328 & 0.290 & 0.167 & 1160 & 1336 \\
\hline & 333 & 0.281 & 0.163 & 1124 & 1304 \\
\hline
\end{tabular}

\section{Mathematical Formulation}

The theory of heat conduction of liquids established a concept in which heat transfer in liquids occur through hydro acoustic vibrations (phonons) in the liquid medium [18]. Based on this Debye's theory of heat conduction of liquids, Bridgeman, Borovik, Cordos, Mamedov and others derived a relations are based on the direct proportionality between thermal conductivity and sound velocity in homogeneous liquids.

$$
\mathbf{k}=\mathbf{a v}
$$

Where $\mathrm{k}$ is the thermal conductivity of the liquid, $v$ is the ultrasonic velocity in the liquid and a is the constant expressed in terms of various physical parameters of the liquids.

Bridgeman's thermal conductivity formula provides accurate results with comparison to conventional values obtained from transient hot wire method, parallel plate heat transfer method and others. Bridgman assumed that liquid molecules are arranged in a cubic lattice and that energy is transferred from one lattice plane to the next at the speed at which sound travels through the liquid. The following is Bridgman's equation:

$$
\mathbf{k}=3\left(\frac{\mathrm{N}}{\mathrm{v}}\right)^{\frac{2}{3}} \mathbf{K v}
$$

In this equation, $\mathrm{N}$ (Avogadro's number $)=6.023 \mathrm{E} 23$ and $\mathrm{V}$ (molar volume $)=\frac{\mathbf{m}}{\mathbf{\rho}} \cdot \mathrm{K}$ (Boltzmann's constant $)=$ $1.3807 \mathrm{E}-23 \mathrm{~J} / \mathrm{K}$. The speed of sound through the liquid is $v$.

It has been reported the usage of Schaaff's theory in finding the thermal properties of Jatropha oil using ultrasonic technique.

The Intermolecular free length is proportional to adiabatic compressibility [19]. Newton-Laplace's relation determined that adiabatic compressibility is inversely proportional to square of ultrasonic velocity. Hence Intermolecular free length can be computed from ultrasonic velocity.

Intermolecular free length $\mathrm{L}_{\mathrm{f}}$ can be calculated using the Schaaff [20] equation:

$$
\mathbf{L}_{\mathbf{f}}=\frac{2 \mathbf{V}_{\mathrm{a}}}{\mathrm{Y}}
$$

Where $\mathrm{V}_{\mathrm{a}}$ is the available volume per mole and $\mathrm{Y}$ is the surface area per mole.

$\mathrm{V}_{\mathrm{a}}$ can be expressed as:

$$
\mathbf{V}_{\mathbf{a}}=\mathbf{V}-\mathbf{V}_{\mathbf{o}}
$$

Surface area per mole Y can be written as

$$
Y=\sqrt[3]{36 \pi N_{0}^{2}}
$$

Where $\mathrm{N}$ is the Avogadro Number and $\mathrm{V}_{\mathrm{o}}$ is the volume at absolute zero temperature. For hexagonal packing, free length can be calculated as per Eyring theory as follows

$$
\mathbf{L}_{h e x}=\left(\frac{2^{0.5}}{N}\right)^{0.3}\left(V^{0.3}-V_{o}^{0.3}\right)
$$


For organic liquids, Intermolecular free length $\left(\mathrm{L}_{\mathrm{f}}\right)$ is approx. $20 \%$ higher than $\mathrm{L}_{\mathrm{hex}}$.

Specific heat capacity of the liquid can be calculated using the thermal conductivity relation by considering the intermolecular free length as its mean free path.

Thermal conductivity in a medium is given by

Specific heat capacity can be formulated as

$$
k=\frac{1}{3} C L_{\mathbf{f}} v
$$

Where $\delta$ is the correction factor of the liquid considered.

$$
c_{p}=\frac{3 \mathbf{k}}{\rho \delta \mathbf{L}_{\mathbf{f}} v}
$$

Table: 3.1 Thermal conductivity values for different liquids at different temperatures for both $2 \mathrm{MHz}$ and

\begin{tabular}{|c|c|c|c|}
\hline Liquids & Temperature (K) & $\begin{array}{c}\text { Thermal } \\
\text { Conductivity k (2MHz) } \\
\text { W/mK }\end{array}$ & $\begin{array}{c}\text { Thermal } \\
\text { Conductivity k (4MHz) } \\
\text { W/mK } \\
\end{array}$ \\
\hline \multirow{5}{*}{ Water } & 313 & 0.646 & 0.656 \\
\hline & 318 & 0.650 & 0.657 \\
\hline & 323 & 0.658 & 0.659 \\
\hline & 328 & 0.658 & 0.660 \\
\hline & 333 & 0.659 & 0.666 \\
\hline \multirow{5}{*}{ Ethylene glycol } & 313 & 0.322 & 0.326 \\
\hline & 318 & 0.321 & 0.319 \\
\hline & 323 & 0.320 & 0.315 \\
\hline & 328 & 0.316 & 0.313 \\
\hline & 333 & 0.310 & 0.312 \\
\hline \multirow{5}{*}{ Glycerin } & 313 & 0.317 & 0.314 \\
\hline & 318 & 0.314 & 0.313 \\
\hline & 323 & 0.309 & 0.312 \\
\hline & 328 & 0.308 & 0.309 \\
\hline & 333 & 0.307 & 0.306 \\
\hline \multirow{5}{*}{ Benzene } & 313 & 0.178 & 0.204 \\
\hline & 318 & 0.175 & 0.198 \\
\hline & 323 & 0.170 & 0.196 \\
\hline & 328 & 0.167 & 0.192 \\
\hline & 333 & 0.161 & 0.187 \\
\hline
\end{tabular}
$4 \mathrm{MHz}$ frequencies

Table: 3.2 Specific heat and Intermolecular free length for water and benzene at different temperatures for

\begin{tabular}{|c|c|c|c|c|}
\hline Liquids & $\begin{array}{c}\text { Temperature } \\
\text { (K) }\end{array}$ & $\begin{array}{c}\text { Inter molecular free } \\
\text { length }\left(\mathrm{L}_{\mathrm{f}}\right) \\
\left(10^{-10} \mathrm{~m}\right)\end{array}$ & $\begin{array}{c}\text { Specific Heat } \\
\left(\mathbf{C}_{\mathrm{p}}\right) \\
(\mathrm{J} / \mathrm{kg} . \mathrm{K})(2 \mathrm{MHz})\end{array}$ & $\begin{array}{c}\text { Specific Heat } \\
\left(\mathrm{C}_{\mathrm{p}}\right) \\
(\mathrm{J} / \mathrm{kg} . \mathrm{K})(4 \mathrm{MHz}) \\
\end{array}$ \\
\hline \multirow[t]{5}{*}{ Water } & 313 & 0.9147 & 4719 & 4705 \\
\hline & 318 & 0.9369 & 4597 & 4598 \\
\hline & 323 & 0.9594 & 4495 & 4493 \\
\hline & 328 & 0.9824 & 4392 & 4317 \\
\hline & 333 & 1.0060 & 4293 & 4295 \\
\hline \multirow[t]{5}{*}{ Ethylene glycol } & 313 & 1.4461 & 1891 & 1891 \\
\hline & 318 & 1.4816 & 1847 & 1847 \\
\hline & 323 & 1.5182 & 1806 & 1804 \\
\hline & 328 & 1.5556 & 1764 & 1760 \\
\hline & 333 & 1.5930 & 1721 & 1723 \\
\hline \multirow[t]{5}{*}{ Glycerin } & 313 & 1.1045 & 1821 & 1821 \\
\hline & 318 & 1.1284 & 1785 & 1783 \\
\hline & 323 & 1.1530 & 1747 & 1747 \\
\hline & 328 & 1.1775 & 1713 & 1714 \\
\hline & 333 & 1.2023 & 1679 & 1681 \\
\hline \multirow[t]{5}{*}{ Benzene } & 313 & 3.5319 & 1439 & 1442 \\
\hline & 318 & 3.6233 & 1410 & 1404 \\
\hline & 323 & 3.7167 & 1356 & 1373 \\
\hline & 328 & 3.8122 & 1345 & 1341 \\
\hline & 333 & 3.9099 & 1313 & 1314 \\
\hline
\end{tabular}
$2 \mathrm{MHz}$ and $4 \mathrm{MHz}$ frequencies 


\section{Result And Discussion}

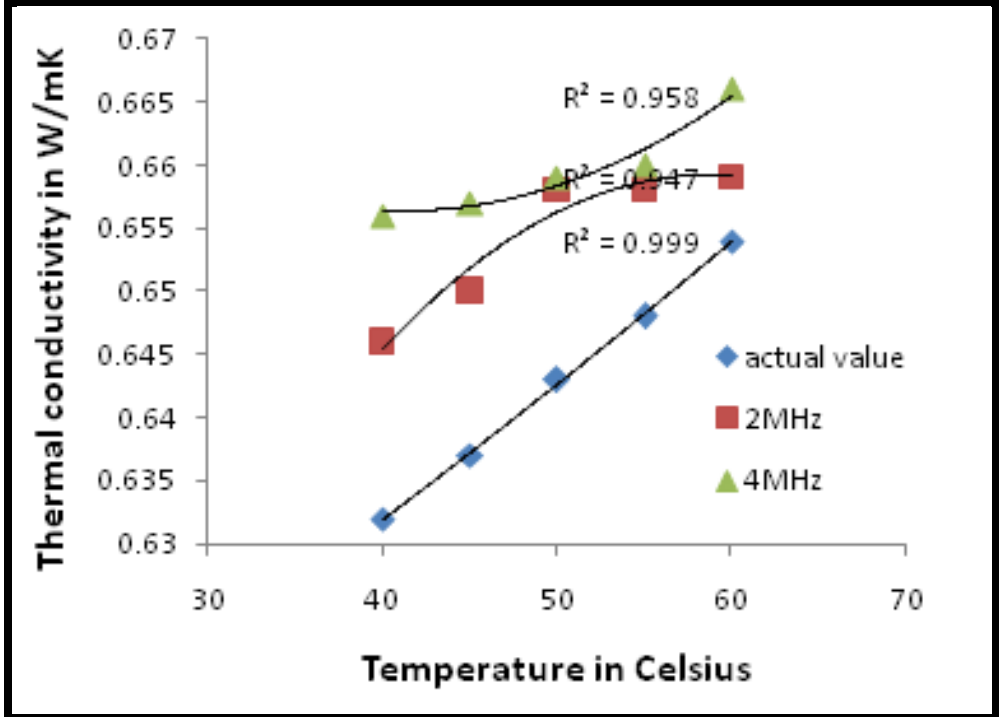

Fig.4.1 Variation of thermal conductivity with temperature for distilled Water

From the Fig.4.1, thermal conductivity for both $2 \mathrm{MHz} \& 4 \mathrm{MHz}$ frequencies of water increases with temperature. However for the same temperature, thermal conductivity of $4 \mathrm{MHz}$ is higher in value compared to 2 MHz. Thermal conductivity depends on how much heat is transferred between the water molecules. $4 \mathrm{MHz}$ frequency imparts more energy by which molecules vibrate at much faster rate. Hence more heat energy travels through the substance, thereby increasing its thermal conductivity and specific heat of the water for the same temperature. It can be observed that $2 \mathrm{MHz}$ frequency provides more accurate results with $0.02(2 \%)$ error values. Increase in frequency increases the risk of obtaining less accurate results. However, It increases the thermal conductivity.

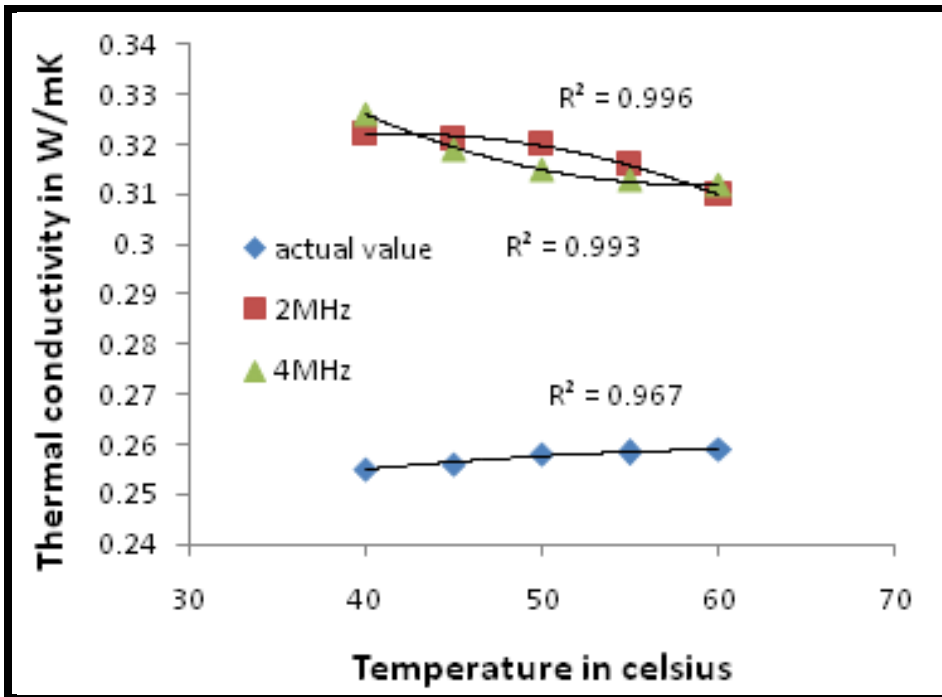

Fig.4.2 Variation of thermal conductivity with temperature for Ethylene Glycol

From the Fig.4.2, Thermal conductivity decreases for both the frequencies with increase in temperature. This provides accurate results to the fact that thermal conductivity of liquids decrease with temperature except for water. As the temperature increases volume of the liquid tends to expand leaving much more gap between molecules. Sound energy which propagates through energy transfer by vibration decreases, making the thermal conductivity to decrease. Thermal conductivity for both the frequencies is nearly the same and intersecting each other. Experimental results differ significantly with the actual values. Slope of the thermal conductivity itself varies as it increases for actual values and decreases for the experimental values. Experimental procedure provides less accurate results for ethylene glycol but it holds good for the theoretical fact of decreasing thermal conductivity for liquids with temperature except for water. 


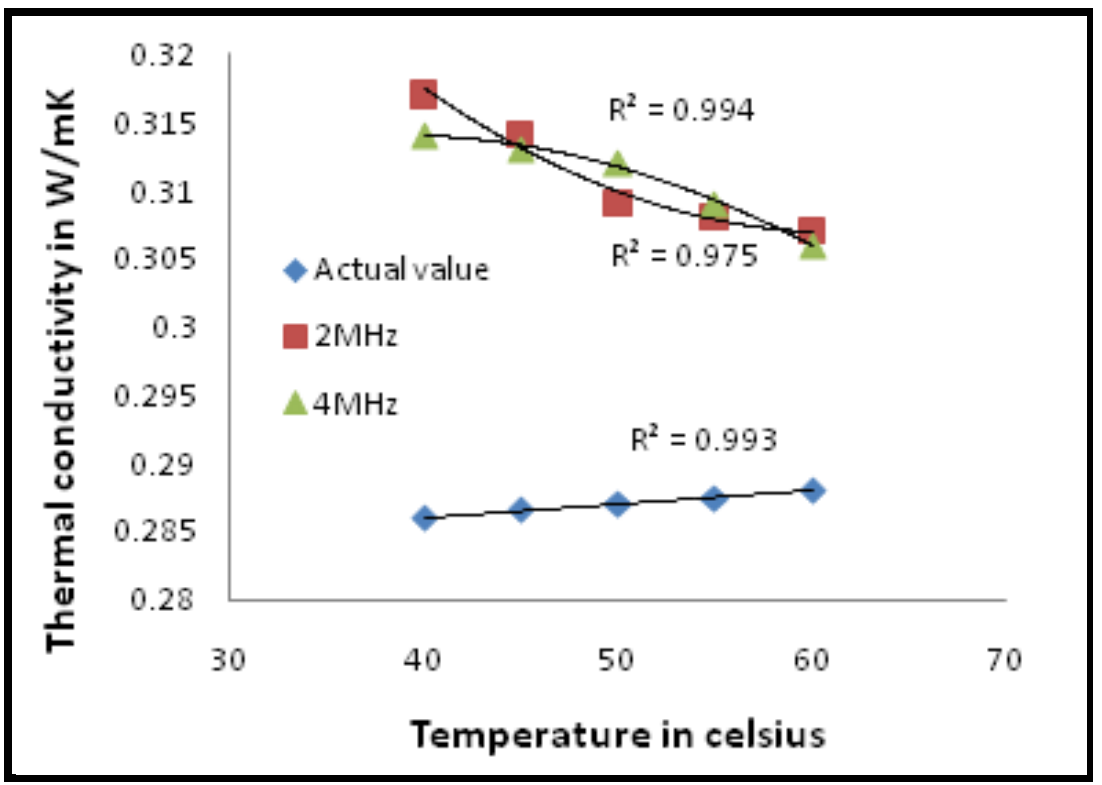

Fig.4.3 Variation of thermal conductivity with temperature for Glycerin

From the Fig.4.3, it can be observed that the thermal conductivity of glycerin decreases with increase in the temperature. Here also the thermal conductivity for both the frequencies is nearly the same and intersecting each other. However for glycerin, thermal conductivity increases with decrease in the frequency of the ultrasonic wave. For $2 \mathrm{MHz}$ frequency, it shows a thermal conductivity of $0.317 \mathrm{~W} / \mathrm{mK}$ at $40{ }^{\circ} \mathrm{C}$, at the same temperature $4 \mathrm{MHz}$ frequency shows a thermal conductivity of $0.314 \mathrm{~W} / \mathrm{mK}$.

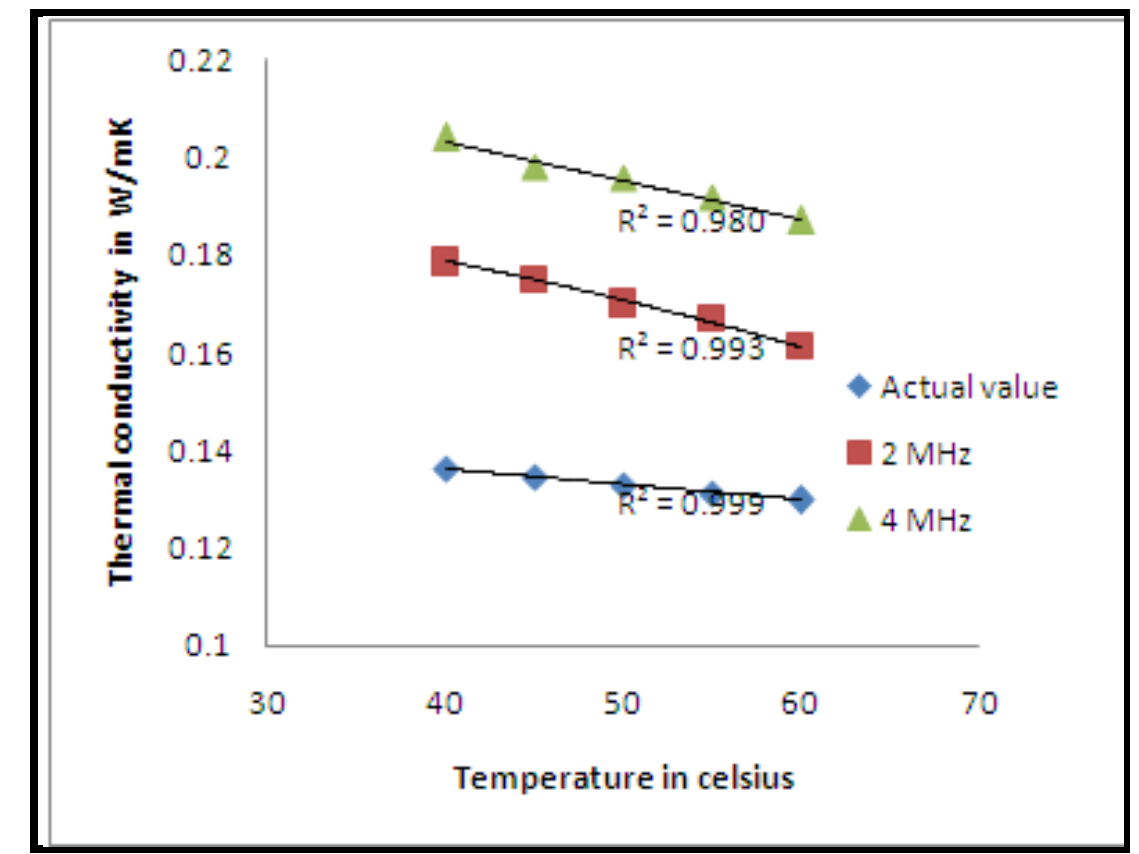

Fig.4.4 Variation of thermal conductivity with temperature for Benzene

From the Fig.4.4, thermal conductivity of benzene decreases with increase in the temperature. Frequency of the ultrasonic wave greatly influences the thermal conductivity of benzene. As the frequency increases from $2 \mathrm{MHz}$ to $4 \mathrm{MHz}$, thermal conductivity increases by 0.02 thus making the liquid equally sensitive to frequency as well as temperature. Thermal conductivity for both $2 \mathrm{MHz}$ and $4 \mathrm{MHz}$ frequencies shows a linear straight line in the Fig.4.4, describing the high rate of accuracy of the calculation. These results provide high accuracy with $0.04(4 \%)$ error in the value calculated. 


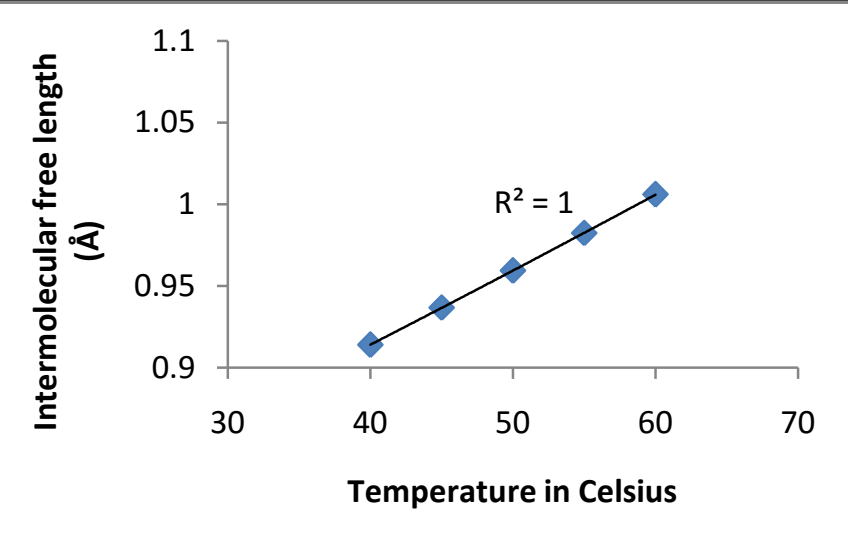

Fig.4.5 Variation of intermolecular free length with temperature for Water

From the Fig.4.5, It can be found that the Intermolecular free length increases as temperature increases. In other words, as temperature increases water molecules gain more kinetic energy and start to occupy more space. As a result, volume of the liquid increases and eventually free length is also increased. Intermolecular free length for water extends more than $1 \AA$.

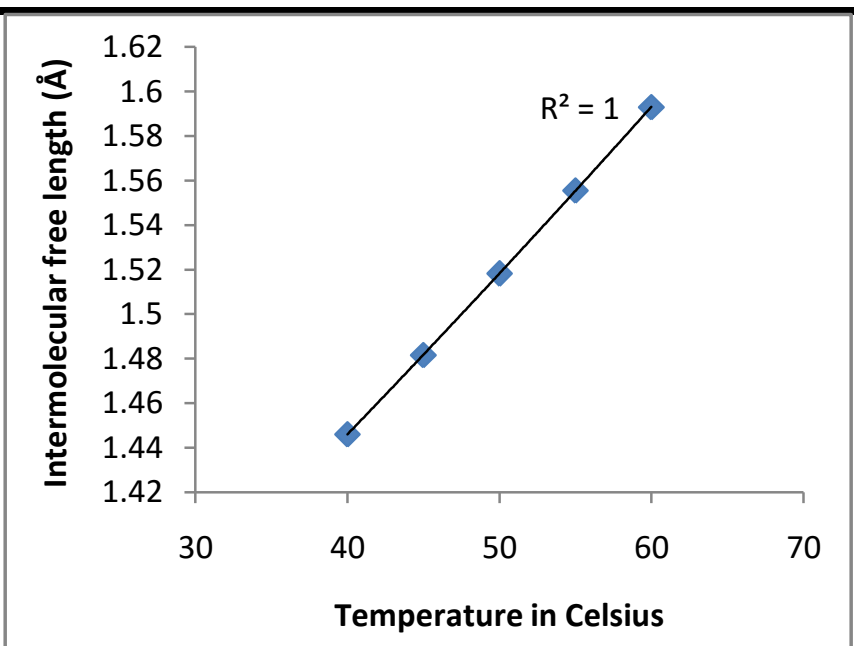

Fig.4.6 Variation of Intermolecular free length with temperature for Ethylene Glycol

From the fig.4.6, Intermolecular free length for ethylene glycol also increases with increase in temperature. However, free length observed is more for ethylene glycol which extend up to more than $1.6 \AA$. Thus ethylene glycol has higher volume as compared to water for same amount of composition.

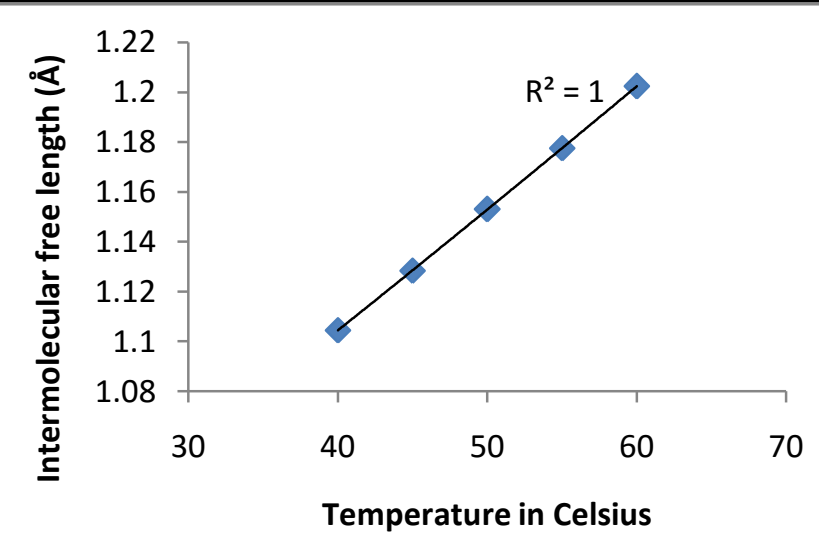

Fig.4.7 Variation of intermolecular free length with temperature for Glycerin 
From the Fig.4.7, It can be observed that increase in temperature increases the intermolecular free length. Free length of glycerin increases linearly with temperature providing accurate value for determination of mean free path of the molecules. Glycerin has a free length of up to $1.2 \AA$ which lies between water and ethylene glycol.

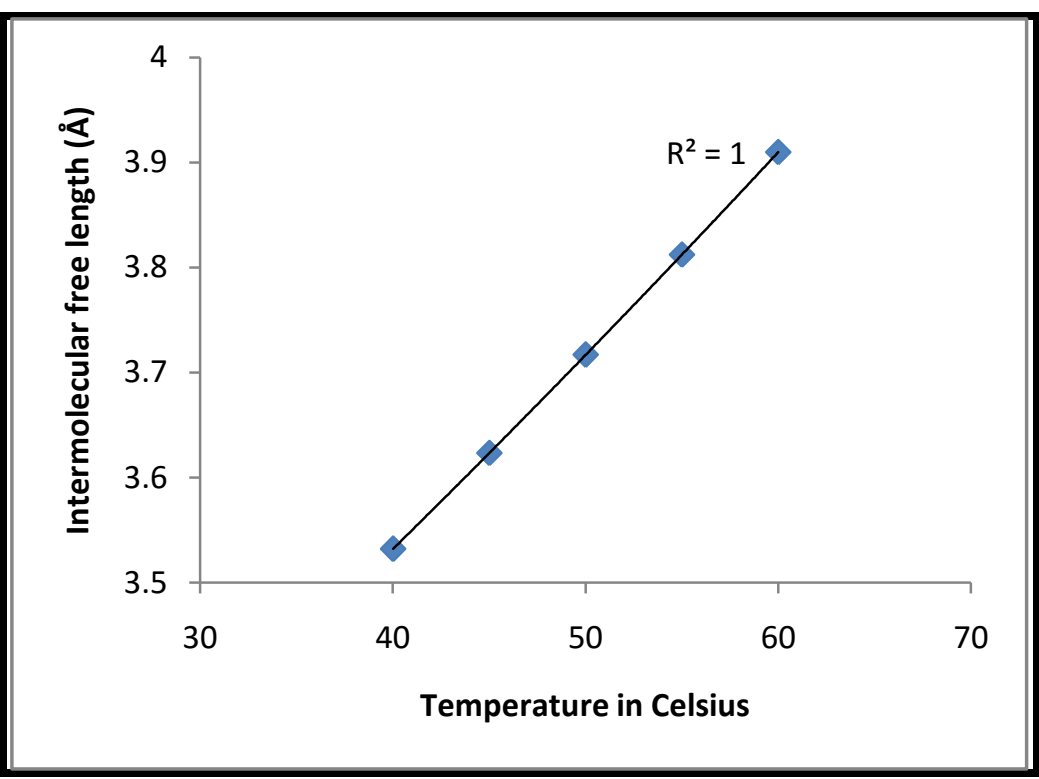

Fig.4.8 Variation of intermolecular free length with temperature for Benzene

From the Fig.4.8, Influence of temperature forms a more linear slope of Intermolecular free length. It also increases with increase in temperature. Free length for benzene is much higher value than for other liquids considered. Benzene has a free length which is nearly $4 \AA$.

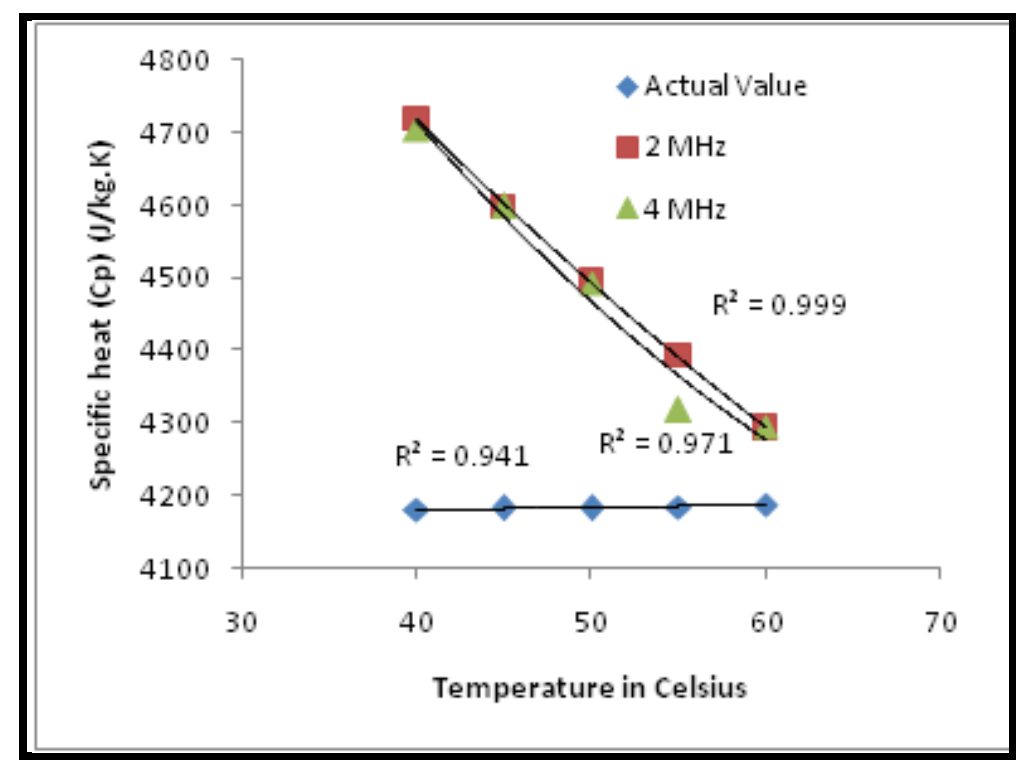

Fig.4.9 Variation of specific heat capacity with temperature rise for Water

From the Fig.4.9, it can be understood that increase in temperature causes the specific heat for both 2 $\mathrm{MHz}$ and $4 \mathrm{MHz}$ frequencies of water to decrease. Decline in the specific heat capacity occurs due to increase in the intermolecular free length. As the free length increases, separation of gap between two molecules increases which restricts the flow of heat energy through the medium and facilitates more energy transfer by radiation. Even though, increase in temperature results in increased specific heat, increase in intermolecular free length causes it to decrease. $\delta=3$ for water which leads to more accurate results. 


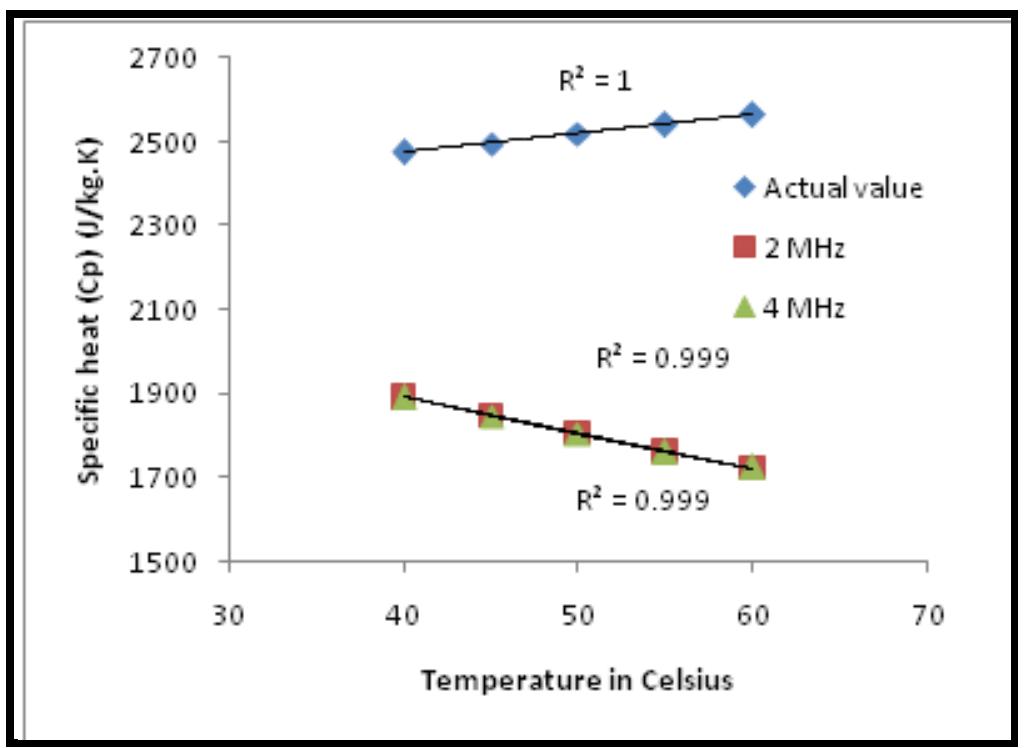

Fig.4.10 Variation of specific heat capacity with temperature for Ethylene Glycol

From the Fig.4.10, specific heat for $2 \mathrm{MHz}$ and $4 \mathrm{MHz}$ frequencies are almost the same. Thus frequency of the ultrasonic wave does not influence its specific heat capacity. Specific heat capacities for ethylene glycol is depend on its thermal conductivity thus decrease in thermal conductivity along with increase in intermolecular free length causes the specific heat capacity to decrease despite the actual condition. $\delta=2$ for ethylene glycol which provides closeness to actual value. It can also be seen that the specific heat capacity for ethylene glycol is nearly one half of the specific heat capacity for water.

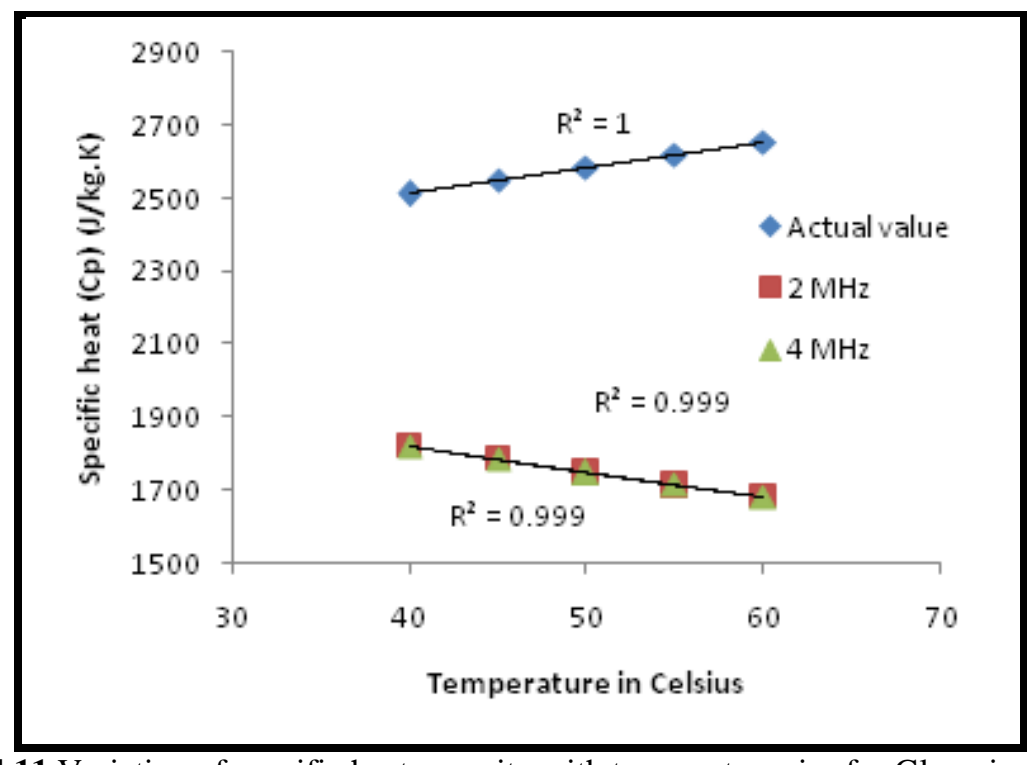

Fig.4.11 Variation of specific heat capacity with temperature rise for Glycerin

From the Fig.4.11, it can also be noticed that specific heat capacity decreases with increase in temperature. This is also due to the fact that increase in intermolecular free length and also the unbalanced forces in the liquid medium causes the specific heat capacity to decrease. It is known that thermal conductivity of liquid decrease with temperature thus specific heat which depends on thermal conductivity also decreases. $\delta=$ 2 for glycerin. 


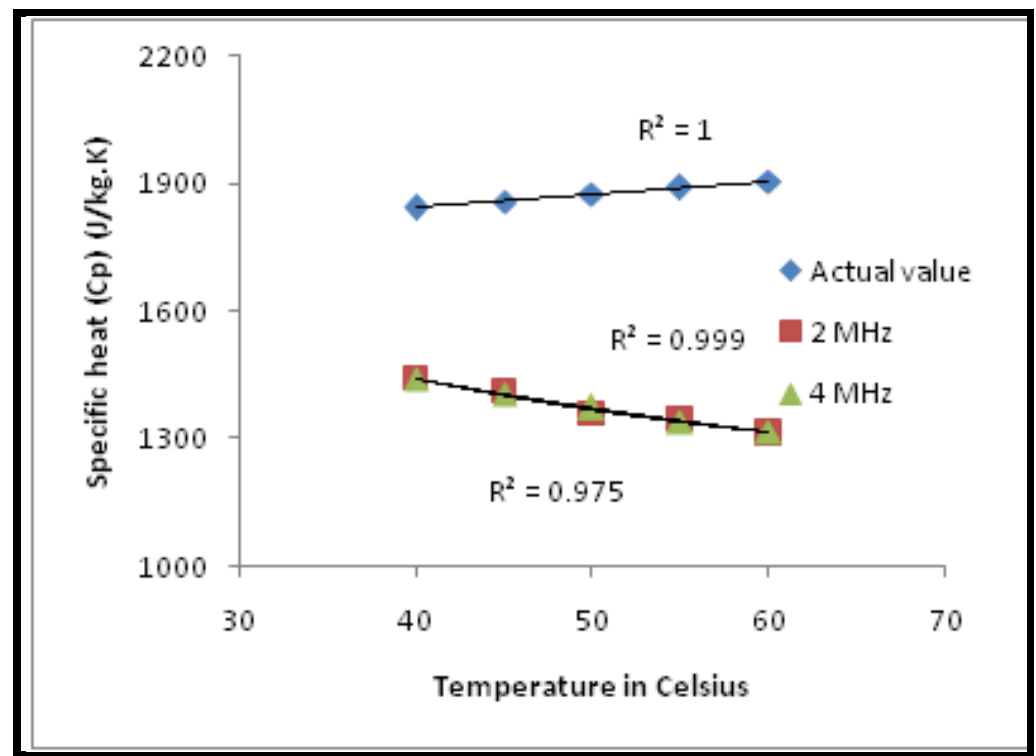

Fig.4.12 Variation of specific heat capacity with temperature rise for Benzene

From the fig.4.12, it can be stated that Benzene having the lower value of specific heat as compared to other liquids considered. Thus it gives more reliable value. Specific heat of benzene also decreases with temperature. Here also influence of frequencies on the specific heat capacity is negligible. $\delta=1$ for benzene.

\section{Conclusion}

Thermal conductivity increase with temperature tends to provide more accurate results for thermal conductivity of liquids which are used in heat transfer applications which are easily susceptible to variable temperature. Influence of frequency of the ultrasonic wave throws light on pressure dependent change of thermal conductivity. Based on the type of the fluid, thermal conductivity either decreases or increases. Ultrasonic wave makes contact with every molecule in the liquid; therefore change in energy of every molecule gets recorded in thermal conductivity calculated. This feature rectifies the error in thermal conductivity calculated using the transient hot wire method which includes density change occurring only nearer to the hot wire in the liquid. Specific heat capacity which is an important thermo physical property used for the selection of liquid for heat transfer purposes can be determined using the free length, thermal conductivity and ultrasonic velocity which are possessed by each molecule. Specific heat capacity decreases as a result of increase in the intermolecular free length which separates the molecules long enough to decrease the ultrasonic velocity and specific heat capacity as well. Ultrasonic technique involving only $2 \%$ error in thermal conductivity calculation for water and the consideration of one thermo physical property as a function of all other thermo physical property enables to provide a more reliable and accurate results.

\section{Notation}

\begin{tabular}{|l|l|}
\hline$v$ & Ultrasonic velocity in $\mathrm{m} / \mathrm{s}$ \\
\hline$\lambda$ & Wavelength in $\mathrm{m}$ \\
\hline $\mathrm{f}$ & Frequency in $\mathrm{Hz}$ \\
\hline $\mathrm{k}$ & Thermal conductivity in $\mathrm{W} /(\mathrm{m} . \mathrm{K})$ \\
\hline $\mathrm{a}$ & Constant in terms of various physical parameters \\
\hline $\mathbf{N}$ & Avogadro number \\
\hline$\rho$ & Density of the liquid in $\mathrm{kg} / \mathrm{m}^{3} ;$ \\
\hline $\mathrm{K}$ & Boltzmann constant in $\mathrm{J} / \mathrm{K}$ \\
\hline $\mathrm{L}_{\mathrm{f}}$ & Intermolecular free length in $\AA$ \\
\hline $\mathrm{V}_{\mathrm{a}}$ & Available volume per mole \\
\hline $\mathrm{V}$ & Volume at the given temperature \\
\hline $\mathrm{V}_{\mathrm{o}}$ & Volume at absolute zero \\
\hline $\mathrm{Y}$ & Surface area per mole \\
\hline$\delta$ & Correction factor \\
\hline $\mathrm{c}_{\mathrm{p}}$ & Specific heat capacity in $\mathrm{J} /(\mathrm{kg} . \mathrm{K})$. \\
\hline
\end{tabular}




\section{References}

[1]. M.Chiesa et.al, "Thermal conductivity and viscosity of water-in-oil nanoemulsions, Colloids and Surfaces A": Physicochem. Eng. Aspects 326(2008) 67-72.

[2]. J.Hemalath et.al, "A novel ultrasonic approach to determine thermal conductivity in CuO-ethylene glycol nanofluids", Journal of Molecular Liquids 197 (2014) 257-262.

[3]. M.Mohammed Nagoor Meera et.al , "On the Thermal properties of Aspartic acid using Ultrasonic Technique", International Journal of ChemTech Research 6(2014) 3685-3689.

[4]. G.Krishna Bama et.al, "A Photoacoustic and Ultrasonic study on Jatropha oil", Journal of Engineering Physics and Thermophysics $83(2010)$

[5]. J.-F. Hoffmann et.al, "Temperature dependence of thermal conductivity of vegetable oils for use in concentrated solar power plants, measured by 3omega hot wire method International Journal of Thermal Sciences 107(2016) 105-110.

[6]. Gianpiero Colangelo et.al, "Thermal conductivity, viscosity and stability of Al2O3-diathermic oil nanofluids for solar energy systems": Energy 95 (2016) 124-136.

[7]. Hosni I. Abu-Mulaweh and Donald W. Mueller et.al, "The determination of the thermal conductivity of fluids", World Transactions on Engineering and Technology Education, (2006) 5-3.

[8]. Y Nagasaka and A Nagashima et.al, "Absolute measurement of the thermal conductivity of electrically conducting liquids by the transient hot-wire method", Journal of Physics E: Scientific Instruments, (1981) 14-12.

[9]. L.E.Kuruzowa et.al, "Determination of thermal conductivity and thermal diffusivity of papaya as a function of temperature", $2^{\text {nd }}$ Mercosur congress on chemical engineering.

[10]. R.B. Bird et.al, Transport Phenomena, John Wiley \& Sons, New York, (2011).

[11]. R. A. Mustafaev et.al, "A formula relating the thermal conductivity of liquids to the sound velocity in them, Journal of Engineering Physics and Thermophysics 7(1997) 27-29.

[12]. J D Pandey et.al, "Intermolecular free length and free volume of pure liquids at varying temperature and pressures", Indian journal of chemistry, 47(2008) 1020-1025.

[13]. Sunanda S.Aswale et.al, "Adiabatic compressibility, intermolecular free length and acoustic relaxation time of aqueous antibiotic cefotaxime sodium", Journal of Chemical and Pharmaceutical research, 4(5) (2012) 2671-2677.

[14]. J Hemalatha et.al, "A comparative study on particle-fluid interactions in micro and nanofluids of aluminium oxide", Microfluid Nanofluid, 10(2011) 263-270.

[15]. Theodore William Richards and Arthur Becket Lamb et.al, "New Methods of Determining the Specific Heat and the Reaction-Heat of Liquids", American Academy of Arts and Sciences, 40(20) (1905) 659-680.

[16]. Biao Ma et.al, "Determination of Specific Heat Capacity on Composite Shape-Stabilized Phase Change Materials and Asphalt Mixtures by Heat Exchange System", Materials 9(2016) 389.

[17]. Allan ferguson. and A. H. Cockett et.al, "Specific Heat of a Liquid at Different Temperatures", Nature 138 (1936) 842-843.

[18]. P.Debye, J. Chem. Phys. 31 (1959) 680-687.

[19]. Bertil Jacobson et.al, "Intermolecular free length in the Liquid state. I. Adiabatic and Isothermal compressibilities", Acta Chemica Scandinavica, 6(1952) 1485-1498.

[20]. W.Schaaffs, Molekularakustik, Springler-Verlag, Berlin, (1963). 